\title{
Translating to the Community (T2C): A protocol paper describing the development of Canada's first social epigenetic FASD Biobank
}

\begin{tabular}{|r|l|}
\hline Journal: & Biochemistry and Cell Biology \\
\hline Manuscript ID & bcb-2017-0278.R1 \\
\hline Manuscript Type: & Article \\
\hline Date Submitted by the Author: & 21-Feb-2018 \\
\hline Complete List of Authors: & $\begin{array}{l}\text { Elias, Brenda SI; University of Manitoba, Community Health Sciences } \\
\text { Hanlon-Dearman, Ana; Manitoba FASD Centre, SSCY Centre } \\
\text { Head, Betty; Cree Nation Tribal Health Centre Inc } \\
\text { Hicks, Geoffrey G.; University of Manitoba College of Medicine, } \\
\text { Regenerative Medicine }\end{array}$ \\
\hline $\begin{array}{r}\text { Is the invited manuscript for } \\
\text { consideration in a Special } \\
\text { Issue? : }\end{array}$ & Fetal Alcohol Spectrum Disorder \\
\hline Keyword: & $\begin{array}{l}\text { Fetal Alcohol Spectrum Disorder, Social Genomic Bio-repository, } \\
\text { Translational Studies, Community and Family Participation, Ethics and } \\
\text { Governance }\end{array}$ \\
\hline
\end{tabular}


Title: Translating to the Community (T2C): A protocol paper describing the development of Canada's first social epigenetic FASD Biobank

Brenda Elias ${ }^{1}$, Ana Hanlon-Dearman ${ }^{2}$, Betty Head ${ }^{3}$, Geoffrey G. Hicks ${ }^{4}$

${ }^{1}$ Department of Community Health Sciences, Max Rady College of Medicine, University of Manitoba, Winnipeg, Canada.

Department of Paediatrics, Max Rady College of Medicine, University of Manitoba, Winnipeg, Canada; Manitoba FASD Centre/FASD Network

${ }^{3}$ Cree Nation Tribal Health Centre, Star Program, The Pas, Canada.

${ }^{4}$ Department of Biochemistry \& Medical Genetics; Regenerative Medicine Program, Max Rady College of Medicine, University of Manitoba, Winnipeg, Canada.

Corresponding Author: Dr. Brenda Elias, Department of Community Health Sciences, Max Rady College of Medicine, University of Manitoba, Winnipeg, Manitoba, Canada. Email: Brenda.Elias@umanitoba.ca 
Abstract: Translating to the Community (T2C) is a social bio-repository designed to advance new diagnostic tools and realign community-clinical processes, with the aim to mitigate the short-and-long term impacts of Fetal Alcohol Spectrum Disorder (FASD), prenatal alcohol exposure, and its co-morbidities and behaviors. In this paper, we describe the evolution of this repository as a new translational partnership to advance a precision medicine approach to FASD. Key to its evolution was a partnership between academic researchers, Indigenous communities, families and a regional diagnostic clinic. We further describe the rationale for social biobanking, the type of banking, ethical engagement of families, communities and clinics, their role in repository design, governance, translation and research activities, types of data collected from families, and how the study data is managed, reported and accessed. The repository design includes biological samples, social-contextual-health survey data, and clinical data (which are linkable to administrative data) from community and clinical cohorts of diagnosed children, children prenatally exposed but not diagnosed, children suspected to have had a prenatal exposure, and related siblings, biological parents, and unrelated children and their parents. From these cohorts and families, potential studies drawing on this data will shed light on various risk factors, social and biological pathways, and service utilization issues, with the aim to implement primary and secondary prevention and intervention strategies.

Key Words [Fetal Alcohol Spectrum Disorder, social genomic biorepository; translational studies, community and family participation, Indigenous, ethics and governance] 


\section{Introduction}

Globally, Fetal Alcohol Spectrum Disorder (FASD), a result of a prenatal alcohol exposure (PAE), is the most common neurodevelopmental condition, exceeding $1 \%$ worldwide with an estimated prevalence ranging from 1\% to 5\% (May et al. 2009; Lange et al. 2017a). Prevalence, however, is often underestimated and difficult to measure due to several reasons. While alcohol exposure is the cause of FASD, individuals with PAE may not develop FASD, as genetic and epigenetic influences may play an important role in that determination (Kobor and Weinberg, 2011). In addition, ascertaining PAE may be very difficult due to subjective reporting of alcohol consumption (Chudley et al. 2005). Stigma and potential child-family social service investigations may deter women from reporting alcohol exposure during the pregnancy (Bell et al., 2016). Delayed assessments may occur due to a patient's age at recognition of PAE and referral, cultural validity of the tests, clinician or clinic preference, test availability, and a later maternal disclosure of PAE or referral (Chudley et al. 2005; Astley 2011), all of which result in FASD underestimation (Roozen et al. 2016). In Canada, the diagnostic process, while comprehensive, is time-consuming, costly, and requires an accessible team of medical, psychological, educational and social specialists (Chudley et al. 2005; Clarren et al. 2011; Cook et al. 2016). The full suite of FASD diagnostic criteria cannot be assessed during infancy. Age at diagnosis tends to range from 3 to 11 years of age, and in some regions even later (Chudley et al. 2005; Cook et al. 2016). Currently, there is no standardized test for adults (Cook et al. 2016). In addition, a definitive neurodevelopmental profile of 
FASD has not yet been established (Lange et al. 2017b). Also lacking are established approaches to assess PAE as a risk for co-morbid conditions (Popova et al. 2016). Yet, an early FASD diagnosis, followed by early interventions, is known to decrease the risk for secondary disabilities, including the severity of these effects (Coles et al. 2000). This diagnostic and access quandary has fueled recent calls to improve diagnosis, prevention, services and support.

In Canada, Federal, Provincial, Territorial and Indigenous governmental and nongovernmental organizations have forged inter-jurisdictional partnerships, collaborations and networks to prevent PAE and FASD, improve early identification of PAE/FASD, progress strategies and develop interventions for related lifelong disabilities. ${ }^{1}$ To address and focus on Indigenous concerns, Canada's Truth and Reconciliation Commission on Indian Residential Schools (2015) (recommendation 33 and 34) recommended improved PAE/FASD prevention, diagnosis and interventions for Indigenous children, families and communities. Federal/Provincial/Territorial (F/P/T) ministers responded and recommended studies to identify and validate FASD screening tools and inform solutions for the social-economic impacts of FASD [See footnote 1].

Aligned with these efforts, our Canada-International research team established a translational research program for personalized risk assessment, early diagnosis, treatment, and long-term planning to mitigate the secondary disabilities of PAE/FASD. A

1 For a description of these partnerships and the FPT recommendations on FASD see the following weblink at http://www.scics.ca/en/product-produit/annex-fetal-alcoholspectrum-disorder-fasd-partnerships-inventory/. 
key feature of this program is the development of a social-bio-repository or social biobank.

Typically, biobanks have evolved to translate basic and clinical research into ideas and products from bench to beside. Along an expanded translational research continuum, biobanks can address the state of a population's health by understanding health and disease at the molecular, cellular, somatic and social/population level with the aim to translate that knowledge to the patient, into practice, and then embedded into routine or practice. Such biobanks can therefore become a critical resource for clinical and public health practice, by moving beyond the bench to bedside and to the population. To that end, such biobanks should include an understanding of the social context and the determinants of health, which may include the social construction and experiences of the risk and disease itself (Murtagh et al. 2011). What is missing from this translation pathway is that the research does not directly translate back to the child, family and communities engaged in the research. To elevate this translational social aspect, we opted for a "social biobank" to understand the social epigenetics of FASD. Our research program entitled "Translating to the Community (T2C): A social epigenetic study of FASD" is directed at 1) validating a PAE/FASD diagnostic code for the early identification of PAE/FASD (including co-morbidities and behaviors) in children, families and communities with a known high PAE/FASD prevalence, 2) facilitating prevention (e.g. education and nutritional supplementation) and earlier interventions by developing new tools for diagnosis, identifying susceptibility and mitigating short-andlong term impacts of FASD through education and support, 3) establishing family, community and system readiness for early diagnosis, prevention, treatment and long-term 
planning, and 4) identifying challenges, particularly around stigma and discrimination, including the potential for intervention and support in affected families and communities. In this paper, we describe T2C's evolution to facilitate and support partnership, participation and long-term research. This paper also highlights the value of building a social biobank, which is a repository grounded and framed by the societal interests of affected or at-risk children, families and communities, governed by a communityuniversity partnership, and supported by community-regional programs and clinics, with the aim to understand and address the social context and the determinants of FASD, which may include the social construction and experiences of PAE risk and FASD itself, including co-behaviors and co-morbidities.

\section{Evolution of T2C}

The first scientific catalyst for this program was the Canadian Israel International Fetal Alcohol Consortium we developed to investigate FASD environmental and genetic risks via genetic, nutritional and socio-economic factors associated with FASD. The goals of the consortium are as follows: 1) to reduce the prevalence and severity of FASD through pioneering research on genetic/epigenetic mechanisms, including those related to nutrition and other environmental factors; 2) to facilitate prevention (which includes nutritional supplementation such as Vitamin A) and earlier interventions by developing new tools for diagnosis, identifying susceptibility, and mitigating short-and-long term impacts of FASD through education and support; and 3) to secure and maintain patient and community engagement to significantly increase diagnosis and intervention strategies 
and reduce the stigma associated with this condition. A key scientific activity of this consortium was the use of experimental animal model studies. We have developed wellestablished frog, mouse and rat models of PAE that reproduce many of the characteristic physical, physiological, and functional deficits of human PAE on brain and biological development. Using these models, we try to understand the mechanisms underlying the outcomes of FASD, including documenting the role of changes in gene expression and epigenetic modifications in the lasting changes in neurobiological/behavioral function observed. Epigenetic signatures, as such, can be the basis of FASD diagnostic tools to facilitate early intervention and to investigate the role of nutrition in reducing the prevalence and severity of FASD.

The second scientific catalyst was the innovative research funded by Canada's Network of Excellence, NeuroDevNet, which led to the recruitment of the single, largest age-andsex matched case-control cohort $(n=110)$ of children diagnosed with FASD (Portales et al. 2016). This study investigated the effects of PAE on genome-wide DNA methylation (DM) profiles via buccal epithelial cells (BECs) of children with FASD. Significantly altered DNA methylation profiles were found. A number of the DM sites and regions showed mirrored genes and pathways previously associated with PAE. Newly discovered sites and regions also involved pathways implicated in FASD functional deficits. In addition, broader patterns related to altered neurodevelopmental disorders (autism and/or epilepsy) were identified within sets and networks of genes associated with FASD. It was also noted that other social-genetic factors could heavily influence the phenotype and outcome. 
All told, this seminal research inspired our efforts to reveal and validate biomarkers of PAE and FASD. Our research therefore builds on groundbreaking studies, as reviewed by Lussier, Weinberg and Kobor (2017), that identified the many alterations in DNA methylation patterns, chromatin states, and ncRNA expression levels associated with FASD and PAE. To advance this research, a more comprehensive approach was required, which promoted biomarker validation and discovery, social environmental assessment, community and clinical readiness, and FASD prevention and intervention studies. The ideal structure to undertake this research was a social-biobank.

Evidence suggests that a biobank is the most appropriate resource to support research in genomics and personalized medicine. By accepting, processing, storing and distributing biological samples and associated data, there is a greater likelihood to enhance our understanding of the biology of PAE/FASD. By adding the social, there is an improved likelihood that we can understand and address the societal and behavioral precursors of PAE/FASD and this lifelong disability. In developing our biobank, we reviewed paediatric and maternal child health biobanks to ascertain the best approach. In the United States, for instance, several state biobanks have evolved from newborn screening programs. The Michigan Neonatal Biobank (http://www.mnbb.org), as an example, collects dried blood samples from nearly every birth since 1984. The lessons learned from newborn screening is that it was initially designed for public health programs. The intent was not to create or sustain a large-scale genomic biobank. Consequently, these types of biobanks lack clear guidelines on consent, are at-risk of creating therapeutic and 
diagnostic misconceptions, and lack participant opt-in or opt-out rights (Tarini and Lantos 2013). While these biobanks are population-wide, they do not collect a range of samples or social epidemiological data required to fully understand the social genomics of a condition or behavior. Biobanks focused on maternal child health have merit but may not contain sufficient social biological information related to a specific condition and may exclude the father's information. For example, in Canada, the Maternal-Infant Research on Environmental Chemicals (MIREC) study, with data collection now complete, is a large biobank of maternal blood, urine, hair, and breast milk, and cord blood and infant meconium. Other data collected includes pregnancy and lifestyle data on mothers and the pregnancy and medical chart data on infants with the aim to assess prenatal and post-natal exposure to multiple environmental chemicals (Arbuckle et al 2013). When compared to neonatal dried blood spot biobanks, this biobank collected a range of biological samples, administered a survey and collected medical chart data on mothers and infants. While this biobank has had relevance for FASD by providing meconium to validate a meconium screening test to assess fetuses heavily exposed to ethanol in utero, the samples and data collected were not sufficiently focused or comprehensive for a social genomic understanding of PAE/FASD and its associated behaviors and conditions. Another major weakness across all these types of biobanks is that they were not linked or engaged via a partnership with affected children, families and communities. Consequently, these types of biobanks are less likely to address, specifically, the societal impacts of a health condition or behavior or are less likely to improve diagnostic services or programs dedicated to improving outcomes for those atrisk or affected by PAE/FASD. At noted earlier, the work of Canada's NeurodevNet in 
developing an FASD cohort had served as a key catalyst for our social biobank. While this cohort was focused on children diagnosed with FASD, the research program was not designed to collect samples or data in a biobank format or fully engage at risk children and affected families and communities via a partnership governance structure. Nevertheless, NeurodevNet's research was a critical catalyst for T2C and the evolution of a social biobank, which is unique to Canada and the world. In the following sections, we describe further our rationale for biobanking, the type of biobanking, ethical engagement of families, communities and clinics, their role in repository's design, governance, translation and research activities, types of data collected from families, and how the study data is managed, reported and accessed.

\section{Social Biobank Considerations}

At the cellular level, genes carry information on what we look like, how we survive, and how we interact with various environments. These milieus can be a womb, family, household, neighbourhood, community or the physical environment, past or present, in which we reside. These environs are not static, but are fluid to turbulent, benign to toxic. At the interface between genes and the environment, epigenetic changes occur, which are regular, natural occurrences expressed as biological markers on DNA that modify gene expression of the underlying gene sequence without changing that sequence. Epigenetics explains how such environments get under our skin and create biological changes, of which some are positive, negative, lifelong, or transgenerational. For the latter, two potential mechanisms may operate. Environmentally induced epigenetic incitement can 
modify parental behaviour or physiology, which in turn can affect an offspring's postnatal environment, thus shaping a child's behaviour through epigenetic effects. Potentially, environmental signals experienced in pregnancy can affect the epigenetics of three generations, the parents, the fetus, and the fetus' germ line cells, which thus illustrate the possibility for succeeding generational health impacts. The sensitivity, resistance or vulnerability of the fetus to alcohol may also be due to maternal and paternal genetic factors (Mead and Sarkar 2014). In the case of FASD, alcohol may not be the sole insult. The epigenome is highly sensitive to a multitude of environmental insults such as other substances, nutritional deficiencies, stress, etc. (Ramsay 2010). As reviewed by Liyanage et al. (2016) and Mead and Sarkar (2014), studies have shown a transgenerational effect, thus suggesting that FASD phenotypes are modifiable through maternal/paternal genetic factors and epigenetic mechanisms. To understand the aetiology of FASD, understanding the location and overlapping milieus of the prenatal environment is therefore required, where potentially permanent imprinting in physiological and behavioural systems can occur during development, thus leading to short or lifelong health impacts. The degree to which PAE affects development therefore depends on maternal and paternal genetic background, health state and wellbeing, the biological parent's lived experience, their nutrition and substance use (alcohol and other substances), historically, at conception, and during pregnancy. The social environment surrounding the fetus, child and parent is also a factor (Thayer and Kaztawa 2011; Kundakovic and Champagne, 2015). Decades of social epidemiological studies have shown that neurodevelopmental and life course health is strongly associated with social disadvantage and the environment in which people reside (Kubzansky et al. 2014). Early 
life programming and neurodevelopmental health further supports this perspective (Bale et al. 2010). As a result, socially informed DNA methylation studies are key to revealing the way in which adverse or positive environments are programmed into human biology. By engaging a social epigenetic approach in a validation study, we will be able to identify and link social and biological disadvantage to social, physical, behavioural or economic milieus. Such an approach requires research tools and processes conceptually informed by the sciences of epigenetics, social epidemiology, clinical epidemiology, and the social science areas of social justice and human rights. By interlinking a multitude of frameworks and tools, we will have more conceptual power and the evidence to thereby rethink the developmental origins of disease, including how best to promote health equity for children, families and communities impacted by PAE and FASD. By engaging families and communities in sufficient numbers and collecting linkable social, biological and clinical data, there is greater power to detect small changes in a vast sea of unrelated cellular noise and to best characterize the issues and gaps as a means to address the needs of clients and to realign services and systems. These considerations informed the type of biobank we created.

\section{Type of Biobank}

By definition, a biobank is a large collection of biological or medical data and tissue samples, gathered for biomedical research purposes. These repositories can be diseaseoriented, population based, or virtual. A disease-oriented biobank usually has a hospital or clinic affiliation and collects biological samples to look for biomarkers affiliated with a 
disease or disorder. Population-based biobanks collect samples for disease susceptibility biomarkers in a general population. A virtual repository, in contrast, is a large database accessed via a web portal or specialized software and can be populated by high-resolution sample images and characterizing data (Hueser et al. 2010; De Souza and Greenspan 2013). Regardless of type, biological samples and/or data are held to share and facilitate research over time and increase opportunities for broader studies, framed by similar or competing perspectives, beyond those possible from a small collection. The other value of a biobank is that it operates at the interface of genomics and society. To operate effectively, bio-repositories generally should include an associated archive of epidemiologic data that are essential for molecular epidemiology and translational studies (Donatella et al. 2008). Basic epidemiological and clinical data are typically collected to meet that goal. For molecular epidemiology, data should contribute to identifying potential genetic and environmental risk factors at the molecular level, with the aim to inform the aetiology, distribution and prevention of disease across populations and within families. For translational studies, data should inform medical practice and meaningful health outcomes. To fully reflect the interface between genomics and society, researchers also need to think beyond the medical setting of the patient (Genome Canada 2017). Biorepositories that include those with the condition, those at risk where exposure is known or suspected, their siblings and parents, and unrelated controls can validate genomic tests, ready the at-risk group for new assessment tools, and frame, during validation, primary and secondary preventions and interventions. A population bio-repository approach also has merit, as it can broaden the societal potential of genomic research. A population biobank, however, is costly to launch and maintain, and in some countries, is not 
favoured or permitted (Hueser et al. 2010). Another weakness is that the data collected may not be sufficiently focused for the health condition or behavior and environment under study or for long-term follow-up if co-morbidities cluster.

For our research program, we adopted a social-bio-repository or social biobank approach, as defined above, where recruitment occurs via community-regional programs and clinics. Cohort recruitment occurs at the sub-geographic level, and the protocol permits expansion regionally, nationally and internationally, using a similar sub-geographic cohort recruitment approach. The repository is designed to hold participant biological samples, social survey data, medical chart abstraction data, qualitative data, all of which are linkable; it also includes the participant's permission to link these datasets, where feasible, to government health and social administrative data. By taking a comprehensive approach, the data collected would address genomic, ethical, environmental, economic, legal and social aspects, as mandated by Genome Canada (2017). A major step to design such a repository was its setting and a community-university partnership and governance process, which is discussed below.

\section{The Setting and Framing of a Community-Clinic-University Partnership and Governance Process}

The Province of Manitoba (Canada) was an ideal site to establish the T2C social biobank. The provincial population is served centrally by the Manitoba FASD Centre and Network, which includes a central provincial diagnostic clinic housed within the newly created 
Specialized Services for Children and Youth Centre (SSCY) which is located in Winnipeg, Manitoba, Canada. The SSCY is a provincial initiative focused on integrating services for children and youth with disabilities and special needs. The Manitoba FASD Clinic is academically linked to the University of Manitoba, Max Rady College of Medicine's Departments of Genetics, Paediatrics and Community Health Sciences. There is also a long-established health and social research relationship with Manitoba's and Canada's Indigenous communities, whereby tribal councils and communities work in partnership with Community Health Sciences' academic researchers to improve the health and social services of their community membership. In addition, the clinical academics associated with this social biobank have worked extensively in establishing national diagnostic standards in FASD and then evolving those standards in partnership with the Canadian FASD Research Network (CanFASD) and internationally (Chudley et al. 2005; Cook et al. 2016). The University of Manitoba also has a state of the art Regenerative Medicine Program with trained laboratory and bioinformatics technicians, including a cadre of affiliated national and international researchers working on the genomics of FASD via animal and human studies through an international FASD consortium (already noted). Laboratory facilities permit the biobanking of specimens in a dedicated repository with refrigeration and ultra-low freezer units, and space is dedicated to the processing of data and samples for storage and analysis, including the testing of samples.

To establish the T2C social biobank at the University, a partnership and governance approach was required. A collaborative partnership bridging the University of Manitoba, 
the Manitoba FASD Clinic and Network, and, via Cree Nation Tribal Health Centre, the affected families and communities, was required to initiate and lead the validation of a new way to diagnosis FASD earlier and to confirm PAE in children. Also, by taking a full spectrum view of FASD, including its comorbidities and behaviours, a T2C collaborative partnership was required to mobilize this knowledge, ready the families, communities and health and social system for prevention and an earlier diagnosis, and to address the lifelong secondary disabilities associated with FASD and PAE.

In designing the social-bio-repository, we therefore needed to find social equilibrium between researcher interests and donors, be they families, service providers, programs, clinics, or the various communities they represent. A human rights framework, grounded in the four basic values of human dignity, autonomy, equality and solidarity (Quinn and Degener 2002), informed how we structured the repository (interactions and practices), as a way to promote positive outcomes (participation, opportunity, non-discrimination, wellbeing) for those impacted by FASD. To advance Indigenous solutions for those impacted by FASD, a counter-hegemonic approach was also required, which emphasized a collectivist and reciprocal approach to research (Castellano 2004; Tuhiwai Smith 1999; Wilson 2009; Battiste and Youngblood Henderson 2000; Schnarch 2004). For instance, by engaging Indigenous communities and families as full partners, we recognized their leadership in validating findings, promoting early diagnosis, framing practical prevention and intervention approaches, and reducing stigma and discrimination. This process evolved as follows. 


\section{Tenets of the T2C Participatory Engagement Process}

At the proposal development stage, we hosted an information workshop of potential community partners (government, health and social service agencies and Indigenous tribal organizations). This workshop was then followed by a series of partner stakeholder meetings to build a mutual research relationship for a repository that reflected the interface between genomics and society (children and adults with PAE/FASD, their families, communities and health-social system providers).

These meetings resulted in support for the repository concept from key tribal and clinical partners. The provincial FASD Medical Director joined as a co-principal investigator and assumed leadership of the clinic recruitment cohort and clinic readiness initiatives. While a regional FASD clinic partnership was important, community-family partners were vital to account for genomic-and-service delivery differences across and within populations and for leading change. Indeed, while we share $99.5 \%$ of our DNA, individuals can differ genetically in disease susceptibility. As a result, a personalized approach to diagnosis and treatment may require a further understanding of genetic architecture. While the ethnicity of participants may capture some of the variation in allele frequencies of the genes underlying a potential clinical trait, the challenge remains as to whether populations can be accurately classified in discrete self-reported/genetic ethnicity groups. Because the distribution of genetic variation can be quasi-continuous with neighbouring geographies (Tishkoff and Kidd 2004), we aimed to recruit, via our partners, participants from distinct ancestry/ethnicity and adjacent communities to inform those analyses. In Canada, 
engaging with Indigenous families and communities at the outset is also critical because they are key to diagnostic referral and outcomes. Two tribal councils, which offer mentoring programs for women who are at risk for consuming alcohol in pregnancy, were identified as potential partners. South East Resource Development Council (SERDC) attended the initial stakeholder meetings and helped design the research proposal to the granting council. Cree Nation Tribal Health Centre (CNTHC) joined after the grant was funded. Both councils provided guidance and oversight for various aspects of the research program.

Further discussions then ensued to frame the social-bio-repository. Several stakeholder meetings were held to develop reciprocity mechanisms for: 1) recruiting and supporting partners, research sites and participants; 2) standardizing data collection, management and access; 3) anticipating or responding to impacts from societally informed genomic scientific advances; 4) mapping, generating and integrating research insights; 5) enriching success and avoiding pitfalls; and 6) ultimately integrating and mobilizing knowledge to enhance practices or policies within and among participating communities. Working with the tribal councils, we conducted a scan of existing programs relevant to families impacted by FASD. With CNTHC and the Manitoba FASD Clinic and Network, we deconstructed the ethical processes required for a social biobank (recruitment, consent, assent, future contact, withdrawal of consent, reporting of secondary variant findings, data handling, and data access), developed a conceptual framework to guide survey measures and the fields for the medical abstraction form, and developed a biological sample data quality and tracking process for the biological samples. By involving tribal 
service organizations and the regional clinic at the outset, we respected the power of Indigenous communities and clinics to control their participation in this research, and reciprocally, we invested in readying a multijurisdictional health and social care systems for a new way to diagnose, intervene and prevent PAE/FASD. Particularly for our indigenous partners, we respected free, informed consent. Article 19 of the United Nations Declaration on the Rights of Indigenous Peoples (2007) requires States to consult and cooperate in good faith with indigenous peoples via their representative institutions to obtain free, prior and informed consent before adopting and implementing legislative or administrative measures that may affect them (article 19). When applied to research, free implies that there is no coercion, intimidation or manipulation in developing and managing the repository. Prior implies that consent is sought sufficiently in advance of any authorization or commencement of activities and respect is shown to time requirements and consensus processes. Informed implies that information is provided. This information may include: the nature, size, pace, reversibility and scope of the proposed project; its purpose, duration, locality and areas affected; preliminary assessment of likely economic, social, cultural or environmental impacts, including potential risks; and, identification of personnel likely to be involved in the project, its executive and procedures, including withholding consent. For this process to succeed, consultation and participation are crucial components (United Nations 2007). Given this obligation to secure tribal council full participation in designing the repository and standardize its processes, we also had to be mindful that the communities they represented were equally as important. In Canada, First Nation communities have the sole authority to provide permission for researchers to enter their community to recruit 
participants. Respecting the tenets of First Nation ownership, control, possession and ownership of information and adhering to reciprocal accountability was key for participant recruitment and community readiness initiatives. For example, while Cree Nation Tribal Health Centre (CNTHC) engaged with researchers in the design of the repository and had the design approved by the centre's governing board, the First Nation communities who receive CNTHC services have the ultimate authority to approve who can conduct research in their community. Thus, after CNTHC board approval, we then met with the First Nation communities serviced by CNTHC. We first met with the Chief and Council members, and once their approval was attained (in principle), we then met with the community health centre to obtain their approval. This two-layered process was key to ensure that the communities were providing free and informed consent and were fully engaged in optimizing validation value of the repository.

\section{Repository Validation and Translation Value}

In genomic research, validation studies provide confidence in DNA results by ensuring that the testing method is robust, reliable and reproducible. The data collected also has the potential to reveal any service gaps and alignment issues experienced by the child, family, diagnostic clinics (e.g., for biomarkers), and FASD support and intervention programs. Such validation value is achieved through governance (as noted) and appropriate cohort design, the collection of a wide range of data and its long-term storage. (See Figure 1) Included in the design are defined procedural limitations, quality control 
and monitoring procedures, and standardized operating procedures and interpretation and publication guidelines. The validation value of $\mathrm{T} 2 \mathrm{C}$ was enhanced in these ways.

To illustrate, our next section describes our cohort subjects and recruitment, followed by other key areas (as noted above) and then we conclude with a section on the power of validation when linked to a translation pathways platform.

Figure 1: Translating to the Community (T2C) Governance and Protocol Structure

\section{INSERT FIGURE HERE}

\section{Cohort Subjects and Recruitment}

For this study, a multidimensional validation cohort of at-risk children, families and controls was deemed ideal for validation and long-term follow-up. Following a review of the literature, and after much discussion and debate, the cohort and control groups identified were as follows, with sample sizes proposed for each to establish the repository, which then will increase when additional sites representing different populations (national and international) are recruited: 1) children with a clinically confirmed FASD diagnosis $(n=600) ; 2)$ children who were prenatally exposed to alcohol but have not been diagnosed with FASD ( $n=600)$; 3) children who have not been diagnosed with FASD, who have an unknown alcohol exposure but may have a neurodevelopmental condition or 
presentation, such as learning, motor skills, language, non-verbal communication, mental health, attention, or behavioral issues $(n=600)$; 4) at least one biological sibling (control) who does not have a neurodevelopmental condition or presentation and has not had a PAE (ideally and where possible close in age and sex matched) $(\mathrm{n}=600) ; 5)$ an unrelated child (age and sex matched control) who has not been prenatally exposed to alcohol and does not have a neurodevelopmental condition or presentation $(n=600)$; and 6$)$ the biological parents (maternal $n=600$ and paternal (if available, $n=300$ ) of the above, and if no parents, a legal guardian to provide information on the child and the environment in which they live.

Participant recruitment was initiated first in Manitoba (Canada) starting in 2018, and the protocol permitted recruitment from other regions, nationally and internationally, providing that regional ethics board permission was obtained. There was no end date set for when recruitment would end. Via the consent form, participants were informed that there was no limit on the length of time we would store their samples and information, and that researchers may keep using these samples and information for research forever, unless the participant decides to withdraw from the research.

Recruitment was designed for clinical and community program settings. Potential participants would be recruited, first, through the Manitoba FASD Clinic and Network located in Winnipeg, a large urban centre, and via Tribal-community programs, such as CNTHC Star Mentorship Program, “Jordan's Principle” program and other communitybased health, social or education programs. The recruitment protocol for these sites was 
designed to expand locally, nationally and internationally providing that ethnical approval was attained in those jurisdictions. Community study investigators (CSIs) were drawn from the participating program and clinic areas, and were designated the responsibility to obtain consent, collect data and ship data to the T2C repository at the Regenerative Medicine Program at the University of Manitoba. A T2C-CSI training program was designed for standardized recruitment, obtaining informed consent, data collection, handling and shipping, and how best to field questions from participants and communities about the study. A standardized recruitment poster and script was developed with the clinic and tribal community study investigators and then reviewed by potential participating communities.

\section{Informed Consent}

Working with CNTHC and the FASD clinic, we designed an informed consent form that was aligned with the principles of free, informed consent. Before contributing any samples or information, participants must complete and sign a consent form. Children, if they wished, also had the right to complete and sign an assent form. Via this form, each adult participant (biological mother, biological father, or legal guardian when children are no longer under the care of their biological parents) received detailed information about the purpose of the social-bio-repository study, its data collection procedures, any risk, discomfort, or potential benefit from participating, permission for future studies, including research linking study data to administrative data via personal identifiers, and follow-up, long-term storage of samples and data, current and future uses of samples, de- 
identification and how personal information and privacy is protected (with a special permission to share de-identified data on children), details on whether study participants would receive study results, and withdrawal of consent. The last three items were given careful consideration and are described below.

\section{Privacy, De-identification and Personal Information}

In developing this repository, several steps were taken to protect participant privacy, particularly involving DNA. Via the consent, participants are informed that DNA is unique to participants, much like a fingerprint, and for that reason there is a possibility that DNA without a name on it could be traced back to a participant. For example, if a participant's DNA was stored somewhere with their name attached (e.g., due to a criminal offence) and those samples were matched to the samples participants donated in this research, the possibility that they could be identified is increased. While the chance of that happening is very small, researchers cannot make guarantees that it would not happen. To protect participants from this happening, T2C researchers will be asked to make every effort to protect confidentiality. They are to sign an oath of confidentiality, agree that they will not try to find out who are the participants and can only publish research findings in a general way. Via the consent form, participants are also informed that if they take part in this study, their personal information recorded on consent forms, contact and data linkage information forms, and other corresponding letters and forms will be kept separate from the samples and information they provided. Assurances are given that protected health information will not be given out except when required by law 
such as a criminal offence or when the participants have given the researchers permission to contact them or when their personal information needs to be linked to the provincial population registry file to undertake studies using administrative data (described further in the data access section). Participants are informed that University of Manitoba ethics and research quality personnel will be permitted to look at sections of their research records, for as long as needed, to maintain ethical standards. For this reason, researchers are not able to guarantee complete privacy to participants. Participants are also informed that in some countries there are no laws that prohibited genetic discrimination, which we illustrate via the following examples. For example, a health insurer might refuse to give insurance to a person who has a greater risk for a disease. Employers may use genetic information to decide whether to hire, promote or terminate workers. Participants are informed that all people, regardless of where they live, might be affected by genetic discrimination. They are also advised that genetic information is also personal health information and that all researchers, consistent with privacy and personal health information laws, must protect that information. Participants are informed that all the samples and information provided are de-identified to ensure privacy. At consent, study numbers unique to the participant and family are assigned, and this key, linking the study numbers to their name, is kept separate from the participant's samples and information. Only the study numbers are recorded on the samples and information. Participants are informed that this key is important for reporting secondary variant findings back to participants, managing their withdrawal of consent if that occurred, facilitating the linkage of study data to provincial administrative data when consent to link is given, and permitting future contact for researchers to seek consent from participants for follow-up 
studies if consent is provided for that contact. The following two sections illustrate the value of this key when fulfilling a critical aspect of genomic research concerning secondary variant findings and withdrawal of consent.

\section{Secondary Variant Findings}

In the informed consent, we delineated the types of information study participants may or not receive depending on the permissions they granted. While findings would be reported generally via newsletters, webpage stories, presentations or publications, we are ethically required to address the case for reporting findings directly to participants. In this study, it was critical to address the issue of "incidental findings" in sequencing (Green et al. 2013), a term coined and traditionally used by the clinical and scientific community, to describe biological test findings that were not related to the primary purpose for the test. Recently, the term 'incidental,' while not intentioned, has run the perceived risk of minimizing the significance of the finding. The term, as applied by the American College of Genetics and Genomics, was viewed as not being appropriate when representing potentially life-changing findings, such as a fatal disease or misattributed paternity or misaligned lineage (undisclosed adoption) (Allyse and Michie 2013). In response, the American College of Medical Genetics and Genomics (ACMG) (Kalia et al. 2017) have proposed the term "secondary findings" or "secondary variants." This term was deemed more reflective of what is and is not a research result in social-genomics research, and better reflected a similar ambiguity in clinical contexts around what precisely is and is not a result representing a clinical point of view. That is, secondary variants are typically 
identified by chance, which means they are not actively searched for. According to Wolf et al. (2008), a variant, where there is strong net benefit for sharing that finding with the participant, can be a condition likely to be life threatening or a condition likely to be grave that can be avoided or ameliorated. A variant discovered could also indicate a significant risk of a condition likely to be grave or serious, where that risk cannot be modified, which offers a possible net benefit to share that finding with the participant. A research participant would unlikely benefit from knowing of a secondary variant indicating a condition that is not likely to be of serious importance. In the course of research, misattributed paternity or misaligned lineage (undisclosed adoption) could be discovered (e.g., family studies) and, as noted, is a secondary variant. This finding would usually fall in an unlikely benefit category, particularly because communicating misattributed paternity or misaligned lineage may carry serious burdens for research participants. That being said, when learning misattributed paternity or misaligned lineage would likely be of health importance to the participants, then its communication, along with the secondary variant that is grave or life threatening or that confers a significant risk for a likely serious condition, may have a possible net benefit. The consent form included a section explaining how we would handle such findings. Participants were informed that they would not automatically receive results, except in rare circumstances. In those circumstances, they had a choice to know about such findings and whether they wanted the researchers to notify their physician and assist with arranging genetic counseling. If they opted to know or not know, they had the right to change their mind. A secondary variant reporting protocol was established to guide researchers if a secondary variant was found. The protocol outlined the researcher and ethics board decision-making, 
review and communication process. In the event that a secondary variant is found, the researcher is to complete a secondary variant report form. This form includes a list of questions specific to the University Health Research Ethics Office, as listed below, which that office may use to guide their decision-making: That is, are the secondary findings material (i.e., do they have significant welfare implications for the participant)? Is the secondary finding based on valid and reliable data? Is there a need to independently corroborate the finding? Can the secondary variant be reasonably and independently confirmed? Is there a need to remove the participant from the study? Does this finding have implications for other members of the participant's genetic family? Has the participant expressed a wish to know/not know about any possible findings? Are the findings able to be treated or ameliorated? Will disclosing the findings create unnecessary anguish or harm for participants? Was the likelihood of uncovering these findings disclosed to participants as part of the informed consent process? Will the participants be able to interpret these findings themselves or will they need assistance (e.g., family physician, genetic counselor) to understand the implications of these findings? How will the finding be communicated to participants taking into consideration their emotional and psychological health? If a secondary variant were found, the project lead would contact the Ethics Officer by confidential email within 48 hours ( 2 business days) of discovering the finding. This email would be followed by the submission of a secondary variant finding report (as noted above) to the University Health Research Ethics Office (Bannatyne Campus) within 72 hours (3 business days) of reporting the finding. The Ethics Officer would then work with the project lead to identify the best course of action for ethics review of the finding. At this stage the identity of the research subject would 
remain confidential. To inform their deliberation, we outlined a process they may follow, which includes considering the scientific and clinical validity of the finding, and whether or not it would be appropriate to disclose the finding to the participant, and confirm if there was participant consent for this disclosure, and determine an appropriate method for disclosing the finding to the participant. If there was an impracticability or impossibility of disclosing such findings to the participants the Project Lead is required to fully describe that issue to the University Health Research Ethics Chair. In this study, "impracticable" referred to undue hardship or onerousness that jeopardized the conduct of the research (sample too large, members likely deceased, geographically dispersed, no permission to contact, or difficult to track). Upon review, the Ethics Officer would discuss with the Ethics Chair the risks and benefits of disclosing the finding to the study participant. From this discussion, a determination would be made and reported back to the researcher on the decision to disclose the finding to the participant, including if there was a plan for communicating the disclosure. In some instances, the Chair may opt for other members or the full Health Research Ethics Committee and/or a clinical expert to review the secondary variant case. Critical to this process was the great care required in deciding how best to report the finding to the study participant. In this protocol, reporting must be ethically justified, reviewed and approved by the University ethics committee. In the event that a communication was warranted, the process may include the following: 1) description of results that may be returned; 2) qualifications of the individual identified to return the results to the participant; 3) timing regarding when the results will be returned; 4) mode of communication to be used; 5) plans for pre- and post-counseling for the participant, if appropriate; 6) if the participant is a minor or an individual of diminished 
consent capacity, description of to whom the findings will be returned; 7) description of plans for allowing participants to withdraw from the study; 8) description of plans for removing a sample in some analyses (e.g., misaligned paternity), if applicable; 9) requests for waiver of informed consent/authorization, if applicable; and 10) a description of the consent process and a copy of the form, that includes a section on returning of secondary variant findings. To notify the participant, we also developed a secondary variant letter to participant and a second variant finding statement of consent.

\section{Withdrawal of Consent}

At consent, participants were informed they have the right to stop participating at any time. They were also informed that if they decide to withdraw from the study, they could not withdraw their samples, data or information from studies that have already occurred or are occurring at the time of withdrawal. The reason why withdrawal of consent was denied in this case was that the researchers already had their permission at that time. To leave the project, participants were instructed to contact the lead investigators or the program coordinator about their decision. A standardized protocol was developed to manage withdrawal of consent. At the initial notification, the study team would quarantine all their samples and study information. A letter would then be sent to the participant listing a number of options for the participants to select and confirm in writing regarding the use and disposal of their unused samples and study information. If the participants failed to return the withdrawal of consent form, the samples and information would remain in quarantine and then destroyed after 30 days of this letter. 


\section{Other Ethical Considerations Addressed at Consent}

In this study, age, sex/gender, ethnicity, and where they and their family live or have lived is collected for compositional and contextual purposes. Age and sex/gender is standardly asked in social-biological studies, and depending on the study and target group, the participant's ethnicity may be asked. From a social perspective, some groups may have experienced greater hardship than others, such as discrimination, poverty, or food security. Some groups may have also lived in areas where there is greater hardship. Where people live or have lived, and the hardship they may have experienced including its over-representation in homogeneous groups (as described by ethnicity), may have also shaped their biology (Kubzansky et al. 2014). Teasing out those nuances, while challenging, are critical in social genomic studies of neurodevelopmental conditions (Bale et al. 2010; Kundakovic and Champagne 2015) like FASD. That being said, communities, specific cultural groups and participants may have concerns about being narrowly characterized with a particular risk factor or health state, which easily occurs in epidemiological studies. For example, a recent systematic review of alcohol consumption during pregnancy and FASD comparing Indigenous populations with the general population reported a high risk in Indigenous populations (Popova et al. 2017). However, consideration was not made that risk (via determinants) only pertains to substrata of that population and does not apply to all Indigenous peoples. While such comparative research is critical for highlighting elevated rates, such comparison without social contextual data can easily run the risk of creating an impression that only or all 
indigenous populations carry this high risk and rate. To address these concerns, an explanation was added to the consent informing participants that researchers may consider ethnicity and place of residence when analyzing the data. Assurances were provided that researchers would not report the precise name or location of where the participants live or have lived and that a review committee (later described in this paper) would review all presentations or publications to ensure communities or participating groups were respectfully portrayed. Those additions to the consent form thus formalized the vigilance required to protect donor interests after data collection.

Another consideration involved data community ownership, control, access, and possess (OCAP) of data reflected within Indigenous research principles (Schnarch 2004). To affirm OCAP, the design team built into the individual participant consent form a statement informing participants that, in addition to the researchers, an indigenous organization representing their interests could hold a copy of a de-identified database to support community program purposes. With participants providing direct, free and informed consent, the tribal council and community secured approval to hold and use deidentified data for programming. While the tribal council could secure access to the combined participating community data, the community could receive data specific to their community.

\section{Repository Materials and Methods}

\section{Survey Instrument}


A survey instrument was designed to address the complexity of FASD from a socialbiological perspective. Questions were directed at the lived experience of children and their parents/legal guardians. The questions asked covered family intergenerational physical and mental health, maternal health and outcomes, maternal, paternal and child health behaviors, and the child's and parent's social, economic, food security, nutritional, mental, spiritual, emotional wellbeing and social support environment. There were also questions on service and program access issues. Additional questions to the survey were informed by the theories of stigma, intersectionality, human rights, disability and social dignity. For instance, a discrimination measure was added to reveal the complex ways in which discrimination and stigma can result in a FASD stereotype (e.g., Williams validated composite discrimination scale (Williams 2006; Link and Phelan 2001; Bos et al. 2013; Pescosolido and Martin 2015). The framework "intersectionality" informed measures to makes transparent the way in which children, adults, families and communities are impacted by FASD via interacting social locations and identities such as gender, ethnicity/race, Indigeneity, ability, and socio-economic status (Hunting et al. 2015). Validated measures for these sub-group characteristics were drawn from Statistics Canada's national surveys. Because racism, patriarchy, class, ableism, and colonialism, when embedded in policies, programs and service delivery agencies, can also shape the stigma and discrimination experienced, questions were added to document this experience. For instance, questions on residential school impacts (unique to Canada Indigenous population) were developed, and to identify contemporary forms of trauma and impacts, a validated composite measure for post-traumatic stress disorder was 
included along with questions on historical and contemporary trauma experiences. A disability framework informed our selection of measures on ability relevant to FASD (physical, cognitive and social relational) to add a practical approach to reduce stigma and discrimination (Prince 2012; Chivers 2007). Indeed, disability frameworks can demonstrate the concrete possibility of changing perceptions and building a positive sense of individual and collective identity for those impacted (Mingus 2011). To identify where change is required, a dignity composite measure informed by a human-social dignity taxonomy was included to identify the social processes that violate or promote dignity, the conditions under which that occurs, who was impacted (positive or negative) and in what way (Spiegelberg 1970; Nordenfelt 2004; Jacobson 2009). A validated measure of social support, used in Statistics Canada national surveys, was also used to identify those families where social support was lacking or available.

\section{Medical Chart Abstraction Form}

Linking the FASD diagnostic process to the survey and biological sample information was critical to link the participant, family and clinical environs critical to understanding risk, circumstance, past and present, referral and diagnostic processes, diagnostic decision making, co-morbidities and behaviors, and follow-up care and referral. In the consent form, participants were asked if a physician or other health provider at the regional FASD clinic saw the child participant. If the child was seen at such a clinic, we asked the parent/legal guardian for consent to access this child's medical records from this clinic. A medical chart abstraction form, building on the CanFASD National Dataform Platform 
Project (https://canfasd.ca/topics/National-Database/) was created to collect information pertaining to the referral process, FASD diagnosis and reevaluation process (if required), other co-occurring diseases or behaviors, screening tests (nutritional, diseases and behaviors), X-rays and medical imaging, medications, and any services recommended or accessed. Because there was a chance that the child may need to be reassessed, we designed the instrument to capture these nuances. Consequently, we notified participants that we might look at these records from time to time to update the information collected and that this could take place for as long as the samples were stored at the laboratory, unless the participant told us to stop. When the child turned eighteen, the research team however was required to contact him or her, if there was permission, and ask for consent to access their adult records.

\section{Biological Samples}

For this study, biological samples included buccal cells obtained by the Isohelix DNA buccal swab kit and tube, saliva from the Oragene DNA saliva self-collection kit and blood drawn by a licenced professional using PAXgene ${ }^{\mathrm{TM}}$ blood DNA tubes and EDTA tubes. Community cohort biological samples were limited to buccal cells and saliva samples, with the option to collect blood at follow-up or at study initiation providing that nursing personnel were available, whereas FASD clinical cohort sampling allowed for all three at study initiation. The determining factor for blood collection at the clinic was that personnel were already trained for standardized blood collection, handling and storage. The maximum blood draw was determined by weight according to accepted paediatric 
clinic research guidelines. ${ }^{2}$ A blood draw from a child weighing 4 pounds (or 1.8 kilograms) was $5 \mathrm{ml}$, and for a child weighting 22 pounds (or 10 kilograms) the draw amount increased to $30 \mathrm{ml}$, and as weight increased from an adolescent to adult, the maximum draw was $34 \mathrm{ml}$. The rationale for collecting of all three types of samples is as follows. While buccal swabs consistently show merit in DNA studies, are less invasive for the patient than blood sampling and may have greater clinical utility, the collection of buccal, saliva and blood will permit a comparative assessment of quality and provide additional samples for future studies on associated conditions.

\section{Standard Operating Procedures for Collection and Storage}

Standard operating procedures were developed to ensure sample and data integrity from collection to storage to retention. For the biological samples, site safety, collection, handling and transportation procedures, unique to each sample extraction type (buccal, saliva and blood) were developed. A quality control tracking form was created to document this process, from collection to processing in the laboratory. Added to the protocol were laboratory procedures for storing and processing and distributing samples for analysis. Since consent also noted that we might create, in the future, a cell line from the blood samples in order to have an unlimited supply for research over an

${ }^{2}$ Blood draws based on charts from the Children's Memorial Research Centre (Chicago, Il) adapted by Rhona Jack, Children's Hospital and Regional Medical Center Laboratory Seattle WA and used by the Committee on Clinical Investigations, Children's Hospital in Los Angles, CA; Baylor College of Medicine, Dallas, TX; Cincinnati Children's Hospital Institutional Review Board, OH; North shore Long Island Jewish Health System; University of California Davis, CA; University of British Columbia, Children's \& Women's Health Centre of British Columbia Research Ethics Board, BC. 
indeterminately long period, a standardized protocol for their creation and management would be required and will be developed prior to cell line development. Standardized procedures also covered how the consents and data instruments were to be collected onsite and the shipped to the Regenerative Medicine Laboratory to ensure privacy and confidentiality and, equally as important, to respect the dignity of the donor families.

\section{Access and Use of Specimens and Data}

To govern access to the data, we formed the T2C Research Committee. This committee enables informed and efficient collaboration, encourages fair, timely and transparent access to data for high quality research, and ensures that access is facilitated in a scientific manner and in accordance with University of Manitoba ethical procedures, whereby participants' rights, interests and privacy is considered paramount. The T2C repository permits access to its data through a formalized application process. To obtain access, applicants must complete a data access application form and attach the required documentation, which includes a research protocol, proof of funding, if available, proof of scientific peer review, if available, decision letter from a research ethics board, the curriculum vita of the principal applicant, and agreement to become a member of the T2C research team. At application, the applicant must list the data they are requesting and what they will return. The data returned should include all data shared including variables derived from the use of the $\mathrm{T} 2 \mathrm{C}$ data, including those derived from other databases. Returned data includes derived variables, which are variables that are created from other variables using an expression. For example, a derived variable can be an index combining 
several variables (e.g. risk scores) or a numeric variable created by doing the sum of values stored in two or more numeric variables. The return of these data could occur in any database compatible format, and a complete data dictionary or codebook must be provided for the datasets returned.

To avoid any misuse or inadvertent disclosure of information that could indirectly identify research participants or disclose their private data to unauthorized individuals, the T2C Research Committee requires that the applicant follow basic security, use and access practices. To access data, the applicant must also become a member of the $\mathrm{T} 2 \mathrm{C}$ research team and adhere to acknowledgment and publication practices. Upon research committee approval, access to the $\mathrm{T} 2 \mathrm{C}$ research data will be granted for the timeframe agreed upon in the data access agreement (starting from the date of signature of the agreement). Successful applicants much complete an annual report to continue access and use of the research data beyond a one-year period. A copy of the University of Manitoba Research Ethics Board Annual Approval Letter must accompany this report. All approved users must agree to permit their name, their institution/unit's name, and a lay and scientific summary of their research added to our publically available study research registry, including having it listed on our webpage. Each applicant must abide by, where applicable, the following security and compliance measures. The applicants must agree to be an affiliated T2C research member and are required to sign an Oath of Participation and Confidentiality Agreement. They must sign, depending on their research, an agreement to access data and/or bio-specimens. Their institution/unit must have a formal IT policy. They must maintain T2C research data in secure physical computer systems. If 
data are stored on portable computers (whether laptops or other mobile devices), the data must be encrypted in order to avoid any potential disclosure in case the portable system is lost or stolen. They must agree to maintain data in a secure environment and that any analyses must be stored in a secure physical computer system. If the data are stored on portable computers (whether laptops or mobile devices), the data must be encrypted in order to avoid any potential disclosure in case the portable system is lost or stolen. The applicants must agree to implement appropriate access security so as to ensure that only the authorized individuals mentioned in Section I of this Data Access Application Form be allowed to access the research data. This requires, for example, that if $\mathrm{T} 2 \mathrm{C}$ research data is stored on a shared computer system or on a file server, that it be password and encryption-protected. Moreover, if the computer(s) that hold $\mathrm{T} 2 \mathrm{C}$ research data are backed up, the backed-up media must also be encrypted and stored in a secure location. For biological sample/data requests, any laboratory personnel working on biological samples must follow appropriate procedures and be authorized individuals as mentioned in Section I of the application form. Applicants acknowledge that any team member who uses the T2C data (mentioned in Section I of this Data Access Application Form) should be trained in the responsible use of participant information and be familiar with the terms and conditions of data access. In addition, the applicants are responsible for transmitting and familiarizing the other research personnel and students with the restrictions on access and use of the $\mathrm{T} 2 \mathrm{C}$ research data. These team members must also be briefed on the security plans as listed below. If the $\mathrm{T} 2 \mathrm{C}$ research data is stored on a network-accessible computer, there should be measures in place to prevent access by computer hackers or contamination by viruses and spyware. Upon completion of their project, they must 
destroy all local copies, including backups of the data accessed from T2C. However, if necessary, they can keep the T2C data for archival purposes in conformity with national audits, publication or legal requirements. As a check, applicants are required to list the date they intend to complete such requirements. They are also required to either store their analysis code or send a copy of it to $\mathrm{T} 2 \mathrm{C}$ committee in case there is a need to reproduce their variables or findings at a later date. Applicants must also acknowledge that if access is granted, any discoveries, presentations, publications or public communications must name and acknowledge the core $\mathrm{T} 2 \mathrm{C}$ research team members listed in the discovery and communication agreement. They must also understand that any discoveries, presentations, publications or public communications must name and respectfully acknowledge the collective contribution of the Indigenous peoples who participated in this research, including the contributions of various agencies, programs and clinics involved. Moreover, they must acknowledge and agree that the T2C research committee reserves the right to review these outputs to ensure privacy and confidentiality and to ensure that the research respectfully represents and acknowledges the Indigenous peoples, the agencies, programs and clinics who participated in this research.

\section{Access for Linking Study Data to Administrative Data}

To improve outcomes for children and families impacted by FASD in Manitoba, we asked Manitoban participants for consent to link their study information to the provincial administrative and clinical databases held at the Manitoba Centre for Health Policy (MCHP). This university centre is a state of the art population research data repository. 
The repository is a comprehensive collection of administrative, registry, survey, and other data relating to residents of Manitoba. The repository holds, under stewardship, records for virtually all contacts with the provincial health care system, and the Manitoba Health Services Insurance Plan (including physicians, hospitals, personal care homes, home care, and pharmaceutical prescriptions) of all registered individuals. The centre also acts as a repository for census data, clinical datasets and other types of administrative (e.g., education, family services, housing, and justice). These records are linkable through a scrambled identifier provided by the provincial ministry to the MCHP. To facilitate data linkage, consenting participants were asked to provide the following personal information as recorded on their provincial health card: the participant's full names, birth date, postal code, 9-digit health number and 6-digit family number. This information, which is stored separate from the study data, can then be accessed for a data linkage study. An additional layer of approvals will be required to access potentially linkable information.

In accordance with provincial laws, researchers are not allowed to personally link this data. Only the provincial ministry, with approval from the university ethics committee and the Manitoba Health Information Protection Committee, can link the participant's personal information to the provincial population registry. Once linked, it is de-identified and a scrambled personal identification number unique to the Manitoba Centre for Health Policy is generated and associated with the participant's T2C study identification number. This unique scrambled number is required to link the de-identified participant's study information to the health and social administrative databases held at the MCHP. 
When a researcher wishes to undertake a study that links $\mathrm{T} 2 \mathrm{C}$ survey data to the provincial administrative data, they would first seek approval first from the T2C Research Committee using the data access application process, as noted in the previous section. During the approval process, the applicants would be advised to vet their study through the MCHP for feasibility, and once deemed feasible by MCHP, they would receive T2C approval if all conditions were met. After this approval, they would then apply for University ethics approval and following that make an application to the Manitoba Health Information Protection Committee to link the study data to the administrative data. When approval is received, the applicants would then have to sign a data agreement with that agency and provide copies of these approvals to the University Ethics Board and the T2C research committee.

\section{Resources and Networks}

As illustrated, a repository has many nuances and process that requires adequate resources and personnel for on-going data collection, services for sample and data processing, storage without interruptions, and maintaining protocol standards. A major challenge, consistent with bio-repositories generally, is the available funds to support the research. The team has been successful in acquiring funding from the Canadian Institutes of Health Research and the Manitoba provincial government to build the research partnerships, set up the repository and start data collection. Ongoing efforts to maintain and expand the repository will occur through new funding initiatives and partnerships, provincially, nationally and internationally. To secure the future of the repository, we 
helped forge the Canada Israel International Fetal Alcohol Consortium (CIIFAC) and we are collaborating with members of NeuroDevNet, a previously funded centre of network excellence in Canada and its new entity that evolved from this network, the Kids Brain Health Network. Members from this consortium and centre of excellence/network are key team members who will be engaged in building the cohorts and enhancing validation, as illustrated in the next section. Via CIIFAC, our team also includes researchers from Hebrew University (Israel) and Reunion Island (France) and discussions, internationally, are underway to recruit researchers and research sites from other countries.

\section{The Power in a Validation and Translation Platform}

Building on our networks and resources via this social biobank, we will extend and apply recently published findings on epigenetic biomarkers for PAE/FASD (Portales-Casamar et al. 2016; Lussier et al. 2018). These findings showed promise that epigenetic markers of PAE and FASD might exist and provided the strongest evidence to date that buccal DNA methylation assays could have clinical utility. It is interesting to note that many of the epigenetic markers identified in these and other studies are associated with genes with known functions in neurodevelopment, metabolism, stress response and behavior, and as such, underscores the importance of understanding the relationship between the impacts of the social environment and FASD outcomes. Moreover, it is important to recognize that epigenetic changes in gene expression can intrinsically be reversed or restored, perhaps even preventing or reducing some FASD outcomes. Key to achieving these potential outcomes is a more advanced understanding of social epigenetic relationships. 
Via the T2C social biobank and the range of samples (buccal, saliva and blood), we aim to extend, confirm, validate and apply these findings, which would address an important knowledge gap and have important implications for translating epigenetic research to health benefits. For these T2C biological sample analyses, DNA methylation analysis will be conducted on samples collected from each subject, according to established protocols. We will also assess genetic variants. We will conduct methylation and genetics analyses to evaluate the impact of potential confounding factors, such as population stratification, to ensure that we are predicting relevant group differences rather than a correlated feature. Given the comprehensive nature of the survey data, additional analyses could assess predictive differences related to the maternal and paternal environment. By linking biological data to survey data, we will be able to build integrative prediction models for PAE and FASD status. Consistent with other biobanks, platforms, testing and analytical approaches will evolve over time, thus providing comparative and innovative analysis opportunities. All of these considerations and opportunities will be managed via our governance structure.

To build on the success of these biomarker validation analyses, we are evolving a translation validation pathway framework to engage FASD clinic teams, diagnostic services personnel and referral providers from federal-provincial FASD programs, regional health authorities, and social service, education and justice forensic agencies. Via this framework, we intend to engage clinic and diagnostic service end-users with the aim to map out this pathway as a critical first step to inform future clinical and diagnostic 
service validation and adoption, improved access to services, and help realign services to take advantage of early diagnosis and intervention and to promote regional care policy changes. By linking end users to validation, such engagement can produce models we can test (in the future) for the adoption of the PAE/FASD code in this province and other provincial and territorial regions. The recruitment of international sites will also extend this model of engagement, with modifications for fit, to those regions. Moreover, the power of translational validation will be accentuated further with increased samples collected at these new sites.

We will also interrogate child and caregiver pathways using our survey and medical record data (referral, diagnostics, service support referrals). Using descriptive and regression analyses, we can describe, compared to controls, the social, health, economic, cultural, nutritional, behavioral and trauma environments of children, families and communities impacted by PAE and FASD. We will also be able to assess the referral and clinical assessment process in the clinic, including the caregiver's access to and experience with FASD risk assessment, referral and the diagnostic process, including their uptake and experience with services, support and referrals after diagnosis. These data and analyses will inform preventions, interventions and the realignment of services and supports for early risk assessment and diagnosis.

\section{Conclusion}


This brief overview of the $\mathrm{T} 2 \mathrm{C}$ social-bio-repository described our efforts to provide a resource for social-genomic-clinical translation research on FASD. The key value of this repository, first and foremost, is the partnership with Indigenous communities and clinics and our joint respect for human rights. The social capital our researchers and partners bring to this research is what makes repositories successful. Following that is the added value of repositories generally. The repository makes available a collection of samples, social-contextual-health survey data, and clinical data, linkable to administrative data, for transdisciplinary translational research. The focus of the repository extends beyond FASD and includes its co-morbidities and behaviours. The recruitment of community and clinical cohorts comprised of diagnosed children, children prenatally exposed but not diagnosed, children suspected to have had a prenatal exposure, and related siblings, biological parents, and unrelated children and their parents enhances the power to identify molecular expressions. From these cohorts and families, potential studies can shed light on various risk factors, social and biological pathways and service utilization issues, with the aim to implement primary and secondary prevention and intervention strategies. The repository can expand into other communities and clinics, locally, nationally and internationally, and this expansion will permit a wide range of investigations to validate biomarker findings across these groups. In conclusion, the partnership process used to develop the T2C social-bio-repository project illustrates the steps required to undertake this work. Academic-community-family-and-clinical partnerships were key and will always remain the foundation for future repositories. While time-consuming, this process led to the development of the various components of this repository, all of which were designed to further the best interests of the participating families, communities and clinics. 


\section{References}

Allyse, M., and Michie, M. 2013. Not-so-incidental findings: the ACMG recommendations on the reporting of incidental findings in clinical whole genome whole exome sequencing. Trends in Biotechnology 31(8): 439-441

Arbuckle, T.E., Fraser, W.D, Fisher, M., Davis, K., Liang, C-L., Lupien, N., Bastien, S., Velez, M.P., von Dadelszen, P., et al. 2013. Cohort profile: The Maternal-Infant Research on Environmental Chemicals Research Platform. Paediatric and Perinatal Epidemiology, 27(4): 415-425.

Astley, S.J. 2011. Diagnosing fetal alcohol spectrum disorders (FASD). In Adubato, S.A. and Cohen, D.E., Prenatal alcohol use and fetal alcohol spectrum disorders: Diagnosis, assessment, and new directions in research and multimodal treatment, Bentham Science Publisher (Ebook)

Bale, T.L., Baram, T.Z., Brown, A.S., Goldstein, J.M., Insel, T.R., McCarthy, M.M., Nemeroff, C.B., Reyes, T.M., Simerly, R.B., Susser, E.S., and Nestler, E.J. 2010. Early life programming and neurodevelopmental disorders, Biological Psychiatry 68(4): 314-319.

Battiste, M., and Youngblood Henderson, J. 2000. Protecting indigenous knowledge and heritage: A global challenge. Saskatoon, SK: Purich Publishing. 
Bell, E., Andrew, G., Di Pietro, N. Chudley, A.E., Reynolds, J.N., and Racine, E. 2016. It's a shame! Stigma against fetal alcohol spectrum disorder: Examining the ethical implications for public health practices and policies. Public Health Ethics, 9(1): 65-77.

Bos, A.E.R., Pryor, J.B., Reeder, G.D., and Stutterheim, S.E. 2013. Stigma: Advances in theory and research, Basic and Applied Social Psychology 35: 1-9.

Brant Castellano, M. 2004. Ethics of Aboriginal research. Journal of Aboriginal Health 1(1): 98-114.

Chivers, S. 2007. Barrier by barrier: The Canadian disability movement and the fight for equal rights, in Smith, M. (ed.) Group politics and social movements in Canada. Peterborough, ON: Broadview Press, 307-328.

Chudley, A.E., Conry, J., Cook, J.L., Loock, C., Rosales, T., and LeBlanc, N. 2005. Fetal alcohol spectrum disorder: Canadian guidelines for diagnosis, CMAJ, 172(5) suppl. S1-S21. doi: 10.1503/cmaj.1040302.

Clarren, S.K., Lutke, J., and Sherbuck, M. 2011. The Canadian guidelines and the interdisciplinary clinical capacity of Canada to diagnosis fetal alcohol spectrum disorder, J Popul Ther Clin Pharmacol., 18(3) e494-e499.

Coles, C.D., Kable, J.A., Drews-Botsch, C., and Falek, A. 2000. Early identification of risk for effects of prenatal alcohol exposure. J Stud Alcohol 61(4):607-16.

Cook, J.L., Green, C.R., Lilley, C.M., Anderson, S.M., Baldwin, M.E., Chudley, A.E., 
Conry, J.L., LeBlanc, N., Loock, C.A., Lutke, J., Mallon, B.F., McFarlane, A.A., Temple, V.K., and Rosales, T. 2016. Fetal alcohol spectrum disorder: A guideline for diagnosis across the lifespand. CMAJ 188(3), 191-197, doi: 10.1503/cmaj.141593.

De Souza, Y.G., and Greenspan, J.S. 2013. Biobanking past, present and future: Responsibilities and benefits. AIDS 27(3): 303-312.

Donatella, U., Monica, N., Aldo, C.P., Cristina, C., Giuseppe, C., Paolo, I.G., Cecilia, L., Paola, M., Michela, P., Barbara, P., Paola, V., Riccardo, P., and Stefano, B. 2009. The CREST Biorepository: A tool for molecular epidemiology and translational studies on malignant mesothelioma, lung cancer, and other respiratory tract disease, Cancer Epidemiol Biomarkers Prev 17(11): 3013-3019.

Genome Canada, GE ${ }^{3}$ LS Research. https://www.genomecanada.ca/en/programs/ge3lsresearch, accessed March 15, 2017

Green, R.C., Berg, J.S., Grody, W.W., Kalia, S.S., Korf, B.R., Martin, C.L., McGuire, A., Nussbaum, R.L., O’Daniel, J.M., Ormond, K.E., Rehm, H.L., Watson, M.S., Williams, M.S., and Biesecker, L.G. 2013. ACMG recommendations for reporting of incidential findings in clinical exome and genome sequencing. Genet Med. 15(7): 585-574.

Hauser, R.M., Weinstein, M., Pool, R., and Cohen, B. (editors) 2010. Conducting biosocial surveys: Collecting, storing, accessing, and protecting biospecimens and 
biodata. National Research Council of the National Academies, National Academies Press.

Hunting, G., Grace, D., and Hankivsky, O. 2015. Taking action on stigma and discrimination: An intersectionality-informed model of social inclusion and exclusion. Intersectionalities: A global Journal of Social Work Analysis, Research, and Practice, 4(2), 101-125.

Jacobson, J. 2009. A taxonomy of dignity: A grounded theory study. BMC International Health and Human Rights 9(3): DOI: 10.1186/1472-698X-9-3.

Kalia, S.S., Adelman, K., Bale, S.J., Chung, W.K., Eng, C., Evans, J.P., Herman, G.E., Hufnagel, S.B., Klein, T.E., Korf, B.R., McKelvey, K.D., Ormond, K.E., Richards, C.S., Viangos, C.N., Watson, M., Martin, C.L., Miller, D.T., on behalf of the AMCG Secondary Findings Maintenance Working Group. 2017. Recommendations for reporting secondary findings in clinical exome and genome sequencing, 2016 update (ACMG SF v2.0): A policy statement of the Amercian College of Medical Genetics and Genomics. Genetics in Medicine 19: 249-255.

Kobor, M., and Weinberg, J. 2011. Focus on: Epigenetics and fetal alcohol spectrum disorder, Alcohol Research and Health, 34(1): 29-37 https://pubs.niaaa.nih.gov/publications/arh341/29-37.pdf 
Kubzansky, L.D., Seeman, T.E., and Glymour, M.M. 2014. Biological pathways linking social conditions and health: Plausible mechanism and emerging puzzles. In Berkman, L.F., Kawachi, I., Glymour, M.M. (Editors). Social Epidemiology (2 ${ }^{\text {nd }}$ Edition), New York: Oxford University Press.

Kundakovic, M., and Champagne, F.A. 2015. Early-life experience, epigenetics, and the developing brain. Neuropsychopharmacology 40(1): 141-153.

Lange, S., Probst, C., Gmel, G., Rehm, J., Burd, L., and Popova, S. 2017a. Global prevalence of fetal alcohol spectrum disorder among children and youth: A systematic review and meta-analysis, JAMA Pediatr, published on line August 21, 2017 doi:10.1001/jamapediatrics.2017.1919.

Lange, S., Rovet, J., and Popova, S. 2017b. Neurodevelopmental profile of fetal alcohol spectrum disorder: A systematic review, BMC Psychol. 23(5): 22. Doi: 10.1186/s40359-017-0191-2.

Link, B.G., and Phelan, J.C. 2001. Conceptualizing stigma, Annual Review of Sociology 27: 363-385.

Liyanage, V.R.B, Curtis, K., Zachariah, R.M., Chudley, A.E., and Rastegar, M. 2016. Overview of the Genetic Basis and Epigenetic Mechanisms that Contribute to FASD Pathobiology. Current Topics in Medicinal Chemistry 17(7): 808-828.

Lussier, A.A., Weinberg, J., and Kobor, M.S. 2017. Epigenetics studies of fetal alcohol spectrum disorder: Where are we now? Epigenomics 9(3): 291-311. 
Lussier A.A., Morin, A.M., MacIsaac, J.L., Salmon, J., Weinberg, J., Reynolds, J.N., Pavlidis, P., Chudley, A.E., and Kobor, M.S. 2018. DNA methylation as a predictor of fetal alcohol spectrum disorder. Clinical epigenetics 10: 5.

May, P.A., Fiorentino, D., Coriale, G., Kalberg, W.O., Hoyme, H.E., Aragón, A.S., Buckley, D., Stellavato, C., Gossage, J.P., et al. 2011. Prevalence of Children with Severe Fetal Alcohol Spectrum Disorders in Communities near Rome, Italy: New Estimated Rates Are Higher than Previous Estimates. International Journal of Environmental Research and Public Health 8(6): 2331-51. doi:10.3390/ijerph8062331

Mead, E.A., and Sarkar, D.K. 2014. Fetal alcohol spectrum disorders and their transmission through genetic and epigenetic mechanisms, Frontiers in Genetics, 5 (article 154): 1-29.

Mingus, M. 2011. Changing the Framework: Disability Justice: Leaving Evidence. Available at: https://leavingevidence.wordpress.com/2011/02/12/changing-theframework-disability-justice/(Accessed: March 3, 2015).

Murtagh, M.J., Demir, I., Harris, J.R, and Burton, P.R. 2011. Realizing the promise of population biobanks: a new model for translation. Human Genetics 130: 333-345.

Nordenfelt, L. 2004. The varieties of dignity Health Care Analysis 12 (2): 69-81.

Pescosolido, B.A., and Martin, J.K. 2015. The stigma complex. Annual Review of Sociology 41: 87-116. 
Popova, S., Shield, K., Mihic, A., Chudley, A.E., Mukherjee R.A.S., and Rehm, J. 2016. Comorbidity of fetal alcohol spectrum disorder: A systematic review and metaanalysis, Lancet, 5, 387(10022): 978-987. Doi: 10.1016/S0140-6736(15)01345-8.

Popova, S., Lange, S., Probst, C., Parunashvili, N., and Rehm, J. 2017. Prevalence of alcohol consumption during pregnancy and fetal alcohol spectrum disorders among the general and Aboriginal populations in Canada and the United States, Eur J Med Genet, 60(1): 32-48. Doi: 10.1016/j.ejmg.2016.09.010.

Portales-Casamar, E., Lussier, A.A., Jones, M.J., MacIsaac, J.L., Edgar, R.D., Mah, S.M., Barhdadi, A., Provost, S., Lemieux-Perreault, L-P., et al. 2016. DNA Methylation Signature of Human Fetal Alcohol Spectrum Disorder.” Epigenetics \& Chromatin 9 (25): 81-101 doi:10.1186/S13072-016-0074-4.

Quinn, G., and Degener, T. 2002. Human Rights and Disability: The current use and future potential of United Nations human rights instruments in the context of disability. Geneva: Office of the United Nations High Commissioner on Human Rights.

Prince, M.J. 2012. Canadian disability activism and political ideas: In and between neoliberalism and social liberalism, Canadian Journal of Disability Studies 1(1): $1-34$.

Ramsay, M. 2010. Genetic and epigenetic insights into fetal alcohol spectrum disorders.

Genome Medicine 2:27 https://doi.org/10.1186/gm148 
Roozen, S., Peters, G.Y., Kok, G., Townend, D., Nijhuis, J., and Curfs, L. 2016. Worldwide prevalence of fetal alcohol spectrum disorder: Systematic literature review including meta-analysis, Alcohol Clin Exp Res. Vol. 40(1): 18-32.

Schnarch, B. 2004. Ownership, control, access, and possession (OCAP) or selfdetermination applied to research: A critical analysis of contemporary First Nations research and some options for First Nations communities. Journal of Aboriginal Health 1(1): 80-95.

Spiegelberg, H. 1970. Human Dignity: A challenge to contemporary philosophy. In R. Gotesky, E. Laszlo (eds). Human Dignity: This century and the next. New York: Gorden \& Beach.

Tarini, B.A., and Lantos, J.D. 2013. Lessons that newborn screening in the USA can teach us about biobanking and large-scale genetic studies. Personalized Medicine 10(1): 81-87.

Thayer, Z.M., and Kuzawa, C.W. 2011. Biological memories of past environments: Epigenetic pathways to health disparities. Epigenetics 6(7): 1-6.

Tishkoff, S.A., and Kidd, K.K. 2004. Implications of biogeography of human populations for 'race' and medicine. Nature Genetics 36: S21-S27.

Truth and Reconciliation Commission of Canada 2015. Truth and Reconciliation Commission of Canada Calls to Action. Winnipeg, Manitoba, Canada, available at: 
http://www.trc.ca/websites/trcinstitution/File/2015/Findings/Calls_to_Action_Eng lish2.pdf [Accessed 11 September 2017]

Tuhiwai, Smith, L. 1999. Decolonizing methodologies: Research and Indigenous peoples. London: Zed Books.

UN General Assembly. 2007. United Nations Declaration on the Rights of Indigenous Peoples. Resolution / adopted by the General Assembly, 2 October 2007, A/RES/61/295, available at: http://www.refworld.org/docid/471355a82.html [accessed 11 September 2017].

Williams, D.R. 2016. Measuring discrimination resource. (Accessed February 17, 2018 https://scholar.harvard.edu/files/davidrwilliams/files/measuring_discrimin ation_resource_june_2016.pdf).

Wilson, S. 2009. Research is ceremony: Indigenous research methods. Winnipeg, MB: Fernwood Publishing. 
Wolf, S.M., Lawrenz, F.P., Nelson, C.A., Kahn, J.P., Cho, M.K., Clayton, E.W., Fletcher, J.G., Georgieff, M.K., Hammerschmidt, D., Hudson, K., Illes, J., Kapur, V., Keane, M.A., Koenig, B.A., LeRoy, B.S., McFarland, E.G., Paradise, J., Parker, L.S., Terry, S.F., Van Ness, B., and Wilfond, B.S. 2008. Managing incidental findings in human subjects research: Analysis and recommendations. J Law Med Ethics 36(2): 219-2011. 
Figure Caption

Figure 1: Translating to the Community (T2C) Governance and Protocol Structure 
T2C FASD Social Biobank Governance

\section{A. T2C Research Ethics Approvals}
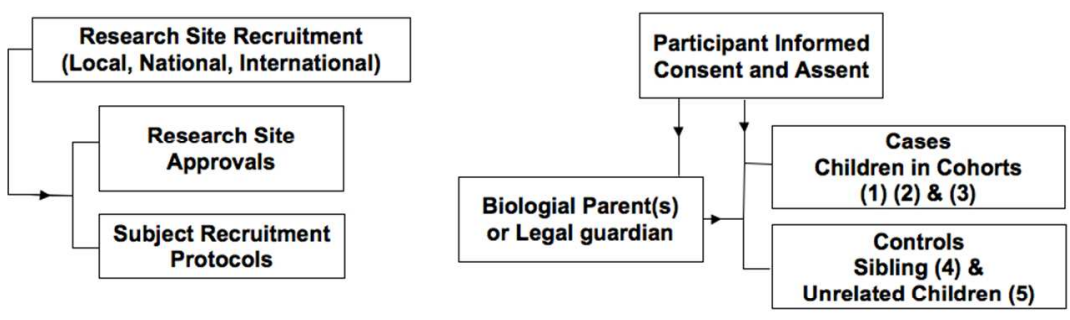

B. T2C Data Collection, Training, Management, Storage Procedures

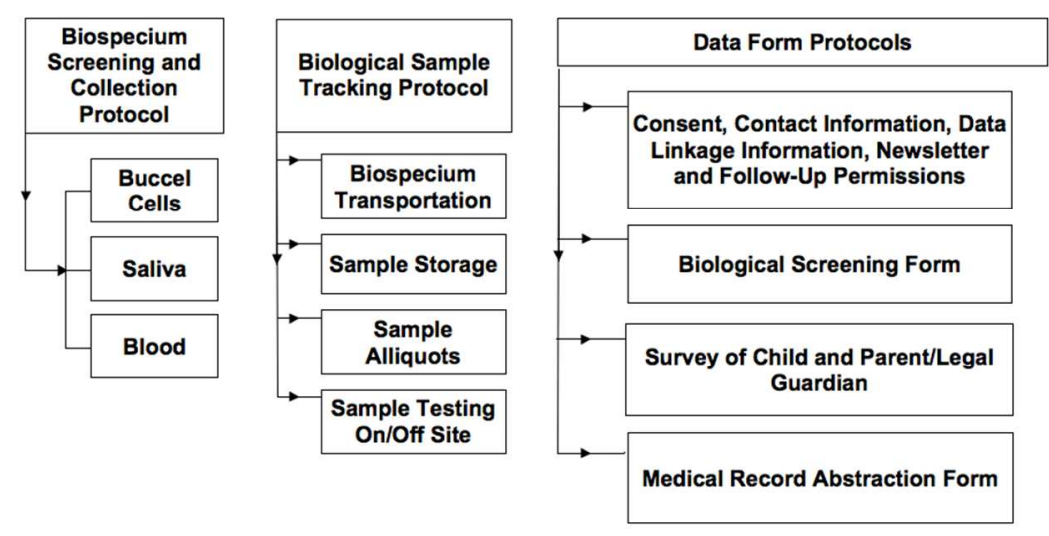

C. T2C Databases, Management, Access, Reporting, Publication and Retention Protocols

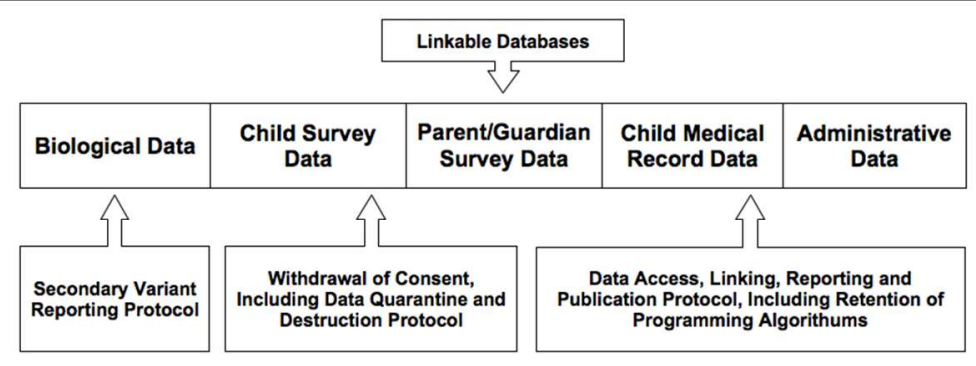

Figure 1: Translating to the Community (T2C) Governance and Protocol Structure $338 \times 478 \mathrm{~mm}(72 \times 72$ DPI $)$ 